\title{
Executive Summary: 2017 ERS/ATS standards for single-breath carbon monoxide uptake in the lung
}

\author{
Brian L. Graham', Vito Brusasco², Felip Burgos ${ }^{3}$, Brendan G. Cooper ${ }^{4}$, \\ Robert Jensen ${ }^{5}$, Adrian Kendrick ${ }^{6}$, Neil R. Maclntyre ${ }^{7}, B_{\text {ruce R. Thompson }}^{8}$ and \\ Jack Wanger ${ }^{9}$
}

Affiliations: ${ }^{1}$ Division of Respirology, Critical Care and Sleep Medicine, University of Saskatchewan, Saskatoon, SK, Canada. ${ }^{2}$ Dept of Internal Medicine, University of Genoa, Genoa, Italy. ${ }^{3}$ Respiratory Diagnostic Center, Hospital Clínic, Institut d'Investigacions Biomèdiques August Pi i Sunyer (IDIBAPS), University of Barcelona, Barcelona, Spain. ${ }^{4}$ Lung Function and Sleep, Queen Elizabeth Hospital, University of Birmingham, Birmingham, UK. ${ }^{5}$ Pulmonary Division, University of Utah, Salt Lake City, UT, USA. ${ }^{6}$ Dept of Respiratory Medicine, Bristol Royal Infirmary, Bristol, UK. ${ }^{7}$ Pulmonary, Allergy and Critical Care Medicine, Duke University Medical Center, Durham, NC, USA. ${ }^{8}$ Allergy, Immunology and Respiratory Medicine. The Alfred Hospital and Monash University, Melbourne, Australia. ${ }^{9}$ Consultant, Rochester, MN, USA.

Correspondence: Brian L. Graham, Division of Respirology, Critical Care and Sleep Medicine, University of Saskatchewan, Saskatoon, SK, Canada, S7N 0W8. E-mail: brian.grahamđusask.ca

@ERSpublications

Summary of updated technical standards for measuring DLCO (TLCO) including the use of rapid gas analyser systems http://ow.ly/TQVG305g3Zq

Cite this article as: Graham BL, Brusasco V, Burgos F, et al. Executive Summary: 2017 ERS/ATS standards for single-breath carbon monoxide uptake in the lung. Eur Respir J 2017; 49: 16E0016 [https://doi.org/ 10.1183/13993003.E0016-2016].

ABSTRACT This document summarises an update to the European Respiratory Society (ERS)/American Thoracic Society (ATS) technical standards for single-breath carbon monoxide uptake in the lung that was last updated in 2005. The full standards are also available online as https://doi.org/10.1183/13993003. 00016-2016. The major changes in these technical standards relate to DLCO measurement with systems using rapidly responding gas analysers for carbon monoxide and the tracer gas, which are now the most common type of DLCO instrumentation being manufactured. Technical improvements and the increased capability afforded by these new systems permit enhanced measurement of DLCO and the opportunity to include other optional measures of lung function.

This article has been revised according to the correction published in the November 2018 issue of the European Respiratory Journal.

Received: Jan 042016 | Accepted after revision: July 242016

This report was approved by the ATS Board of Directors in August 2016 and endorsed by the ERS Science Council and Executive Committee in September 2016. The full version of these standards is available as https://doi.org/10.1183/ 13993003.00016-2016.

Support statement: This report was supported by the American Thoracic Society (grant: FY2015) and the European Respiratory Society (grant: TF-2014-19). Funding information for this article has been deposited with the Open Funder Registry.

Conflict of interest: None declared.

Copyright OERS 2017 


\section{Background}

A standardised clinical method to determine the diffusing capacity of the lung for carbon monoxide (DLCO), using a tracer gas to determine both the alveolar volume and the alveolar concentration of carbon monoxide at the beginning of breath-holding, was described by OGILviE et al. [1] in 1957. This method used the collection of discrete exhaled gas samples from which gas concentrations were measured, using gas analysers that took up to several minutes to perform the measurements. In the remainder of this document we will term these systems and calculations to be "classical". The instrumentation for DLCO measurement has advanced considerably since then, primarily through the use of rapidly responding gas analysers (RGAs) which have a $0-90 \%$ response time of $\leqslant 150 \mathrm{~ms}$. While RGAs are capable of real time, continuous gas analysis, most modern systems generally use this advanced instrumentation only to simulate the classical collection of discrete gas samples in a bag and discard most of the sampled gas data. However, there are several aspects of DLCO measurement that can be improved markedly using all of the data from the continuous measurement technology.

The full technical standards [2], which are published online, are an update of the 2005 European Respiratory Society (ERS)/American Thoracic Society (ATS) standards [3] which, in turn, build upon previous standards $[4,5]$. This update reflects the consensus opinions of both of these societies and is designed to: 1) provide an update to the standards required for DLCO systems based on RGA development; and 2) provide new calculation standards that incorporate continuous gas analysis of the entire exhaled sample. It is recognised that classical equipment will remain in use for some time; however, some previously designed DLCO systems can be upgraded and reengineered to meet these new standards for RGA systems. It is expected that as new DLCO systems are designed and built, they will meet and, in many cases, exceed these new standards.

\section{Methods}

An application was submitted for a joint ERS/ATS task force to update the 2005 DLCO standards [3] with a particular view to systems using RGAs. The task force co-chairs were approved by the ERS and the ATS while the task force members were scientists and physicians with experience in international guidelines, clinical experience of routine lung function testing and specialist knowledge of gas transfer (including research publications).

Potential conflicts of interest were disclosed and vetted. The task force consisted of five members of the task force for the 2005 DLCO standards and four new members. A search using PubMed for literature published between 2000 and 2015 containing various terms related to diffusing capacity and transfer factor yielded 3637 citations. Task force members reviewed the abstracts and identified 113 as relevant to the project and a further 99 as potentially relevant. All manufacturers of pulmonary function equipment used to measure DLCO were sent a survey requesting equipment specifications. Eight of the 13 manufacturers responded. A survey of DLCO equipment specifications published on the manufacturers' websites was also conducted. Using the 2005 standards as a base document, revisions and additions were made on a consensus basis. The recommendations in this document represent a consensus of task force members in regard to the evidence available for various aspects of DLCO measurement (as cited in the document) and reflect the expert opinion of the taskforce members for areas in which peer-reviewed evidence was either not available or incomplete. The task force also identified areas and directions for future research and development where evidence is lacking.

\section{Gas analysers and general equipment \\ Equipment requirements}

The equipment specifications and performance standards for DLCO equipment are summarised in table 1.

\section{Flow and volume analysers}

Any error in measuring flow and subsequently calculating volume will produce a corresponding equal error in DLCO. However, with the continuing improvement in flow measurement technologies, improved accuracy is being achieved. Flow measurement accuracy over the range -10 to $+10 \mathrm{~L} \cdot \mathrm{s}^{-1}$ must be within $\pm 2 \%$. For calibration with a 3 - $\mathrm{L}$ syringe (with a specified maximum error of $\pm 0.5 \%$, i.e. $2.985-3.015 \mathrm{~L}$ ), the calibration volume must be within $\pm 2.5 \%$ which is equivalent to an error tolerance of $\leqslant 75 \mathrm{~mL}$.

\section{Gas analysers}

Rather than collecting a physical sample of exhaled gas, nondispersive infrared carbon monoxide RGAs are used to construct a virtual gas sample from flow and gas concentration data. However, in the signal from such analysers there is both a lag time (the time taken for sampled gas to travel through the sampling tube to the analyser chamber) and an analyser response time (the time taken to reach $90 \%$ of the actual measurement from the time the gas sample reaches the analyser) to be considered (figure 1). The gas concentration signal must be precisely shifted in time to align with the flow signal. 
TABLE 1 Equipment specifications and performance standards

DLco System

\section{Specification}

Required

\begin{tabular}{ll}
\multicolumn{2}{c}{ Specification } \\
\hline Required & Recommended
\end{tabular}

\section{Rapid gas analyser systems}

Analyser specification

$0-90 \%$ response time (see figure 1 )

Maximum nonlinearity

Accuracy

Interference from $5 \%$ carbon dioxide or $5 \%$ water vapour

Drift for carbon monoxide

Drift for tracer gas

Flow accuracy

Volume accuracy (3-L syringe check)

Barometric pressure sensor accuracy

Ability to perform a QA check (3-L syringe; ATP mode; inhaling $~ 2 L$ test gas)

\section{Sample and store data with adequate resolution}

Monitor and report end-expiratory tracer gas and carbon monoxide concentrations (alert operator if washout is incomplete)

Compensate for end-expiratory gas concentrations prior to test gas inhalation in the calculation of $V_{A}$ and $D$ Lco

Ensure proper alignment of gas concentration signals and the flow signal

Measure anatomic dead-space using the Fowler method (see figure 3)

Display a graph of gas concentration versus expired volume to confirm the point of dead-space washout and report the amount of manual adjustment if done (see figure 2)

Measure $V_{A}$ using all of the tracer gas data from the entire manoeuvre in the mass balance equation

Report the $D \mathrm{Lco}$ adjusted for the change in $\mathrm{PAO}_{2}$ due to barometric pressure Ability to input simulated digital test data and compute $D$ LCo, $V_{A}, T L C, V_{D}$

Report the DLco adjusted for the change in $\mathrm{PAO}_{2}$ due to $P_{\mathrm{ACO}}$, if the carbon dioxide concentration signal is available $\leqslant 150 \mathrm{~ms}$

$\pm 1 \%$ of full scale

Within $\pm 1 \%$ of full scale

$\leqslant 10 \mathrm{ppm}$ error in [CO]

$\leqslant 10 \mathrm{ppm}$ over $30 \mathrm{~s}$

$\leqslant 0.5 \%$ of full scale over $30 \mathrm{~s}$

Within $\pm 2 \%$ over the range

of -10 to $+10 \mathrm{~L} \cdot \mathrm{s}^{-1}$

Within $\pm 75 \mathrm{~mL}$

Within $\pm 2.5 \%$

Calculate total volume $\left(V_{A}\right)$ of

$3 \pm 0.3 \mathrm{~L}$ and $D \mathrm{LCO}$ of

$<0.5 \mathrm{~mL} \cdot \mathrm{min}^{-1} \cdot \mathrm{mmHg}^{-1}$ or

$<0.166 \mathrm{mmol} \cdot \mathrm{min}^{-1} \cdot \mathrm{kPa}^{-1}$

Digitise at $\geqslant 100 \mathrm{~Hz}$ per channel Digitise at $1000 \mathrm{~Hz}$ with $\geqslant 14$ bit resolution Implemented ${ }^{\#}$

Implemented ${ }^{\#}$

Implemented ${ }^{\#}$

Implemented ${ }^{\#}$

Implemented ${ }^{\#}$

Implemented ${ }^{\#}$

Implemented ${ }^{\#}$

Calculate values within $2 \%$ of actual values Implemented ${ }^{\#}$

\section{Classical discrete sample systems}

Analyser specification

Maximum nonlinearity

Accuracy

Interference from $5 \%$ carbon dioxide or $5 \%$ water vapour

Drift for carbon monoxide

Drift for tracer gas

Flow accuracy

Volume accuracy (3-L syringe check)

Ability to perform a QA check (3-L syringe; ATP mode; inhaling $\sim 2$ test gas) $\pm 1 \%$ of full scale

Within $\pm 1 \%$ of full scale

$\leqslant 10 \mathrm{ppm}$ error in [CO]

$\leqslant 10 \mathrm{ppm}$ over $30 \mathrm{~s}$

$\leqslant 0.5 \%$ of full scale over $30 \mathrm{~s}$

Within $\pm 2 \%$ over the range of -10

$$
\text { to }+10 \mathrm{~L} \cdot \mathrm{S}^{-1}
$$

Within $\pm 75 \mathrm{~mL}$

Calculate total volume (VA) of 3 $\pm 0.3 \mathrm{~L}$ and $D \mathrm{LCO}$ of

$<0.5 \mathrm{~mL} \cdot \mathrm{min}^{-1} \cdot \mathrm{mmHg}^{-1}$ or

$<0.166 \mathrm{mmol} \cdot \mathrm{min}^{-1} \cdot \mathrm{kPa}^{-1}$

DLCO: diffusing capacity of the lung for carbon monoxide; [CO]: carbon monoxide concentration; QA: quality assurance; ATP: ambient temperature, pressure and humidity; $V \mathrm{~A}$ : alveolar volume; $P_{\mathrm{AO}_{2}}$ : alveolar oxygen tension; $P_{\mathrm{ACO}_{2}}$ : alveolar carbon dioxide tension; TLC: total lung capacity; VD: dead-space volume. \#: Implemented means that the manufacturer has implemented the designated functionality in the DLCo system.

RGA response time

The response time of the RGA will determine how accurately it is able to track the true carbon monoxide and tracer gas concentrations. Even after the application of an appropriate time shift to correct for the lag 


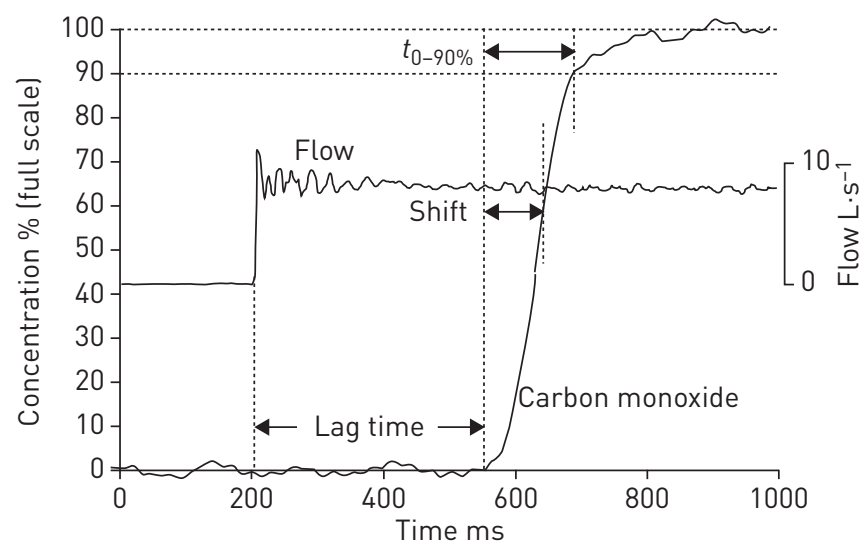

FIGURE 1 Lag and response times for carbon monoxide: the response time of the analyser was estimated by rapidly switching the gas being sampled from zero to full-scale carbon monoxide. The change in the flow signal shows the time at which the switch was made from medical air to test gas. The lag time, the $0-90 \%$ response time and the optimal shift are calculated from the resulting response curve.

and response times, there will be a residual error in DLCO due to the finite response time. For every $100 \mathrm{~ms}$ increase in the $0-90 \%$ response time, the error in DLCO increases by about $0.7 \%$ [6]. Based on the above considerations, the $0-90 \%$ response time for RGAs used in DLCO systems must be $\leqslant 150 \mathrm{~ms}$.

\section{Linearity and accuracy}

The linearity of the gas concentration signals is of primary importance for measuring DLCO, since ratios of gas concentrations are considered in the classical calculations [7, 8]. The error in DLCO measurement due to nonlinearity in the gas concentration signals depends on the size of the lungs and the rate of uptake of carbon monoxide. A nonlinearity of $0.5 \%$ of full scale can cause errors ranging from $0.5 \%$ in a subject with a DLCO of $13.4 \mathrm{mmol} \cdot \mathrm{min}^{-1} \cdot \mathrm{kPa}^{-1}\left(40 \mathrm{~mL} \cdot \mathrm{min}^{-1} \cdot \mathrm{mmHg}{ }^{-1}\right)$ to $1.7 \%$ in a subject with a DLCO of $3.35 \mathrm{mmol} \cdot \mathrm{min}^{-1} \cdot \mathrm{kPa}^{-1}\left(10 \mathrm{~mL} \cdot \mathrm{min}^{-1} \cdot \mathrm{mmHg}^{-1}\right)[6]$. The manufacturer specification for analyser linearity is that any nonlinearity must not exceed $0.5 \%$ of full scale once zero and full scale values have been set.

The accuracy of the gas analyser signal becomes important when measuring the residual alveolar carbon monoxide concentration and the washout of tracer gas from the previous DLCO manoeuvre. The output of a gas analyser must be accurate to within $\pm 1 \%$ of full scale.

\section{Drift}

The gas analyser should have only minimal drift in zero and gain, such that output is stable over the test interval. Gas analyser drift must be $\leqslant 10 \mathrm{ppm}$ over $30 \mathrm{~s}$ for carbon monoxide and $\leqslant 0.5 \%$ of full scale over $30 \mathrm{~s}$ for tracer gas.

\section{Digitisation}

In order for the digitised signal to accurately track the gas concentration signal and to provide adequate opportunity for signal processing for data alignment, the minimum signal sampling rate must be $\geqslant 100 \mathrm{~Hz}$ per channel; however, a rate of $1000 \mathrm{~Hz}$ is recommended.

\section{Other equipment considerations}

Circuit resistance must be $<1.5 \mathrm{cmH} \mathrm{H}_{2} \mathrm{O} \cdot \mathrm{L}^{-1} \cdot \mathrm{s}^{-1}$ up to $6 \mathrm{~L} \cdot \mathrm{s}^{-1}$ flow. If a demand-flow regulator is used on a compressed test gas cylinder, the maximal inspiratory pressure required for $6 \mathrm{~L} \cdot \mathrm{s}^{-1}$ inspiratory flow through both circuit and valve must be $<9 \mathrm{cmH}_{2} \mathrm{O}$.

The equipment dead-space volume (VDequip) for both the inspired test gas and the alveolar sample must be known and their role in all data-computation algorithms should be identified and documented. For adults, the VDequip must be $<200 \mathrm{~mL}$ including the breathing circuit proximal to the gas analyser sampling point, filter and mouthpiece. Smaller dead-space volumes are recommended for paediatric applications and people with a vital capacity (VC) of less than $2 \mathrm{~L}$.

\section{Equipment calibration and quality control}

The considerations for equipment calibration are illustrated in table 2 and are summarised as follows:

1) Flow and gas analysers must be zeroed prior to each manoeuvre. After each manoeuvre, a new zeroing procedure must be carried out to account for analyser drift during the previous test. 
TABLE 2 Equipment calibration schedule

Calibration technique

Frequency

Flow analyser zeroing

Gas analyser zeroing

Volume calibration check

Biologic control

Calibration syringe $D$ Lco check

Calibration syringe leak test

Linearity check (calibration syringe or simulator)

Before each test

Before/after each Test

Daily

Weekly

Weekly

Monthly

Monthly

$D\llcorner c o$ : diffusing capacity of the lung for carbon monoxide.

2) Each day, prior to testing, there must be a volume calibration check with a 3-L syringe [9]. The syringe should be discharged at least three times to give a range of flows varying between 0.5 and $12 \mathrm{~L} \cdot \mathrm{s}^{-1}$ (with 3 - $\mathrm{L}$ injection times of $\sim 6 \mathrm{~s}$ and $\sim 0.5 \mathrm{~s}$, respectively). The volume at each flow must meet an accuracy requirement of $\leqslant 2.5 \%$ error. For devices using disposable flow sensors, a new sensor from the supply used for patient tests must be tested each day. The calibration check may need to be repeated during the day if ambient conditions change. Newer systems monitor ambient conditions and make adjustments as necessary or produce a calibration alert when needed. Older systems may require a calibration check if room temperature changes by more than $3{ }^{\circ} \mathrm{C}$ or relative humidity changes by more than $15 \%$ (absolute). Operators should also perform a calibration check whenever they notice significant discrepancies between inspired volume $(V \mathrm{I})$ and $\mathrm{VC}$, or between alveolar volume (VA) and total lung capacity (TLC), which might suggest volume calibration problems.

3) Each week, or whenever problems are suspected, the following procedures must be followed. First, for those DLCO systems using a volume-type spirometer, a spirometer leak test should be performed according to the manufacturer's specifications. Secondly, a DLCO test should be performed with a calibrated 3-L syringe by attaching the syringe to the instrument in the normal patient test mode. The syringe is then emptied, filled with $3 \mathrm{~L}$ of test gas and then emptied into the mouthpiece after the $10 \mathrm{~s}$ breath-hold. The calculation of VA must be within $300 \mathrm{~mL}$ of $3 \mathrm{~L}$ times the ATPD to BTPS (body temperature, ambient pressure, saturated with water vapour conditions) correction factor, which is $310 / T \mathrm{~A} \cdot P \mathrm{~B} /(P \mathrm{~B}-47)$, where $T \mathrm{~A}$ is the ambient temperature in degrees kelvin and $P \mathrm{~B}$ is the barometric pressure in mmHg. It should be noted that a $3 \mathrm{~L}$ calibration syringe will have an additional dead-space which, depending on the connection to the mouthpiece, is typically $\sim 50 \mathrm{~mL}$ and must be considered in the VA calculation. The absolute value of the calculated DLCO must be $<0.166 \mathrm{mmol} \cdot \mathrm{min}^{-1} \cdot \mathrm{kPa}^{-1}$ or $<0.5 \mathrm{~mL} \cdot \mathrm{min}^{-1} \cdot \mathrm{mmHg}^{-1}$. Thirdly, a test should be performed on a "standard subject" (biological control) or simulator [10]. Standard subjects are nonsmokers who have been found to have a consistently repeatable DLCO (e.g. healthy laboratory personnel). If the DLCO in a standard subject varies either by $>12 \%$ or by $>1 \mathrm{mmol} \cdot \mathrm{min}^{-1} \cdot \mathrm{kPa}^{-1}$ $\left(>3 \mathrm{~mL} \cdot \mathrm{min}^{-1} \cdot \mathrm{mmHg}^{-1}\right)$ from the mean of previous values, the test must be repeated. A study of the long-term intersession variability of DLCO has found that biological control deviations of $>12 \%$ or $>3 \mathrm{~mL} \cdot \mathrm{min}^{-1} \cdot \mathrm{mmHg}^{-1}$ from the average of the first six tests indicate that the instrument is not within quality control limits and must be carefully evaluated before further patient testing [11].

4) Each month a leak test of the 3-L calibration syringe should be performed. If the calibration syringe does not have a volume scale on the shaft, mark $50 \mathrm{~mL}$ below full by measuring the excursion of the shaft from 0 to $3 \mathrm{~L}$ and marking it at a distance that is 0.017 of the full excursion. Fill the syringe and place a stopper at the syringe input. Push the syringe in to the $50 \mathrm{~mL}$ mark (which generates a pressure of about $17 \mathrm{cmH}_{2} \mathrm{O}$ ), hold for $10 \mathrm{~s}$ and release. If the syringe does not return to within $10 \mathrm{~mL}$ of the full position, it should be sent for repair. The procedure is then repeated starting with the syringe at $50 \mathrm{~mL}$ below full, applying the stopper and pulling the syringe to the full position.

5) Each month, gas-analyser linearity should be assessed. A straightforward approach is to measure known serial dilutions of the test gas [12], or to measure the concentration of a separate high-precision test gas having a certificate of analysis. For systems with independent measurements of carbon monoxide and tracer gas, the analyser linearity may also be assessed by comparing the ratio of carbon monoxide and tracer gas concentration to arbitrary dilutions of test gas with room air. A third type of calibration syringe test, which differs from the volume and DLCO checks above by using the 3-L syringe in ambient temperature, pressure and humidity (ATP) mode, may also reveal problems with analyser linearity. With approximately $1 \mathrm{~L}$ of air in the syringe, the test begins by filling the remaining volume with test gas. Following a $10 \mathrm{~s}$ "breath-hold", the syringe is then emptied. The calculation of VA must be within $300 \mathrm{~mL}$ 
of $3 \mathrm{~L}$ with the syringe dead-space being used for the anatomic dead-space in the VA calculation. The absolute value of DLCO must be $<0.166 \mathrm{mmol} \cdot \mathrm{min}^{-1} \cdot \mathrm{kPa}^{-1}$ or $<0.5 \mathrm{~mL} \cdot \mathrm{min}^{-1} \cdot \mathrm{mmHg} \mathrm{m}^{-1}$. A review of quality control data for four different DLCO systems between 2006 and 2015 using this procedure found only four outlier points where $|D \mathrm{LCO}|$ was $>0.13 \mathrm{mmol} \cdot \mathrm{min}^{-1} \cdot \mathrm{kPa}^{-1}\left(0.4 \mathrm{~mL} \cdot \mathrm{min}^{-1} \cdot \mathrm{mmHg} \mathrm{mH}^{-1}\right)$. The same data showed that $V A$ was consistently within $3 \pm 0.3 \mathrm{~L}$ for four systems (unpublished data from B.R. Thompson). Gas mixing in the syringe can be improved by using low flow rates and extending the breath-hold time. The effects of incomplete mixing in the syringe can be minimised by using a larger sample volume. In the absence of a DLCO simulator and high-precision test gases, system checks must be performed using a 3-L calibrating syringe in ATP mode. Manufacturers must provide this test option, which will be the same as the usual testing procedure for a patient, with the exception that VA will be reported under ATP rather than BTPS.

6) A record of equipment checks and standard subject tests should be dated and kept in a laboratory log book or digital file folder. Manufacturers are encouraged to provide software and test equipment options for quality control measurements and quality control data management. In addition, manufacturers may provide equipment-specific, quality-control measures in addition to the foregoing points. If water vapour permeable tubing is used to either remove water vapour or equilibrate water vapour with room air, such tubing must be replaced according to manufacturer recommendations to ensure that it is functioning properly. Chemical gas analyser cells will have a replacement schedule. Manufacturers may also have preventative maintenance schedules for various other system components (e.g. balloon valves) which will require testing and replacement as necessary.

\section{Standardisation issues in the single-breath testing technique}

The single-breath determination of DLCO involves measuring the uptake of carbon monoxide from the lung over a breath-holding period. To minimise variability as much as possible, the following specifications for the standardisation of testing techniques are provided.

\section{Patient condition}

Before beginning the test, the manoeuvres must be demonstrated and the subject carefully instructed. The subject must be seated comfortably throughout the test procedure. The test must be performed at a stable, comfortable temperature within manufacturer's equipment specifications.

\section{Inspiratory manoeuvres}

Once the mouthpiece and nose clip are in place, tidal breathing must be carried out for sufficient time to ensure that the subject is comfortable with the mouthpiece and that the nose clips and mouthpiece are used appropriately with no leaks. The DLCO manoeuvre begins with unforced exhalation to residual volume (RV). In obstructive lung disease, where exhalation to RV may require a prolonged period, a reasonable recommendation is that this portion of the manoeuvre must be limited to $<12 \mathrm{~s}$. Exhalation times of up to $12 \mathrm{~s}$ will allow most patients with airflow obstruction to exhale sufficiently so that they can achieve a maximal VC during the subsequent inhalation of test gas. Submaximal inhalation occurs most frequently in patients with airflow obstruction who are not given adequate time to exhale prior to the inhalation of test gas. At RV, the subject's mouthpiece is connected to a source of test gas, and the subject inhales rapidly to TLC. It is important that the inspired volume of test gas, $V \mathrm{I}$, be as close to the known $\mathrm{VC}$ as possible and $\mathrm{VI}$ must be at least $90 \%$ of the largest $\mathrm{VC}$ in the same pulmonary function testing session. However, a manoeuvre may be deemed to be acceptable if $V_{I}$ is within $85 \%$ of the largest VC and $V \mathrm{~A}$ is within $200 \mathrm{~mL}$ or $5 \%$ (whichever is greater) of the highest VA among acceptable DLCO manoeuvres.

\section{Washout and sample collection}

For RGA systems, the point of dead-space washout can be determined from the expired tracer gas concentration data using an objective algorithm. The beginning of the alveolar plateau can be located by determining the breakpoint of each phase of the washout (a plot of concentration versus volume) and adding a proportion of the dead-space volume measured by the Fowler technique [13] to the phase II to III breakpoint [14]. Such an approach can be automated. For visual verification of the point of dead-space washout, the tracer gas concentration must be displayed as a function of volume, since using the concentration-time curve can be deceptive because of the higher relative flow at the beginning of exhalation (figure 2). If the sample collection point is changed by the operator, it must be recorded in the database and on the report.

With RGA systems, the concentrations of carbon monoxide and tracer gas in a virtual alveolar gas sample can be calculated for use in measuring DLCO; however, RGA systems are capable of simulating much smaller gas samples although it should be noted that such smaller virtual samples will be more subject to noise in the expired carbon monoxide concentration signal. Indeed, JonEs and Meade [15] used gas 
a)

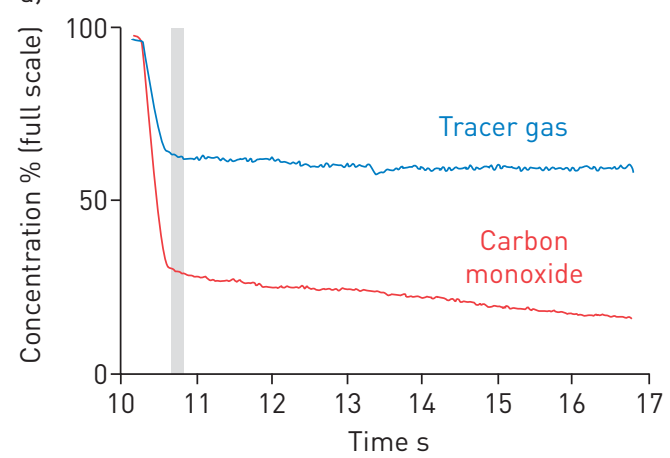

b)

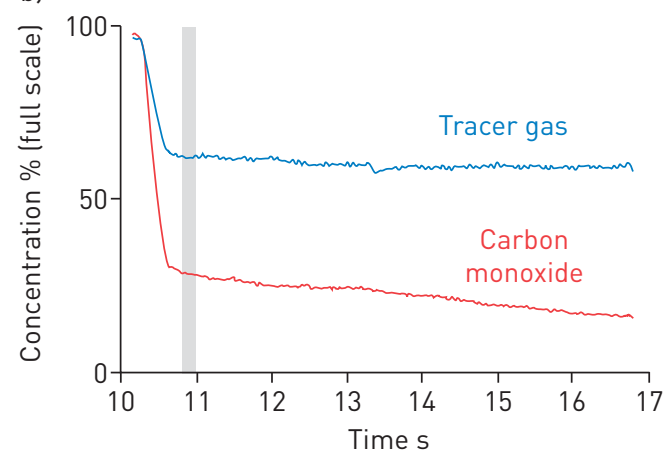

c)
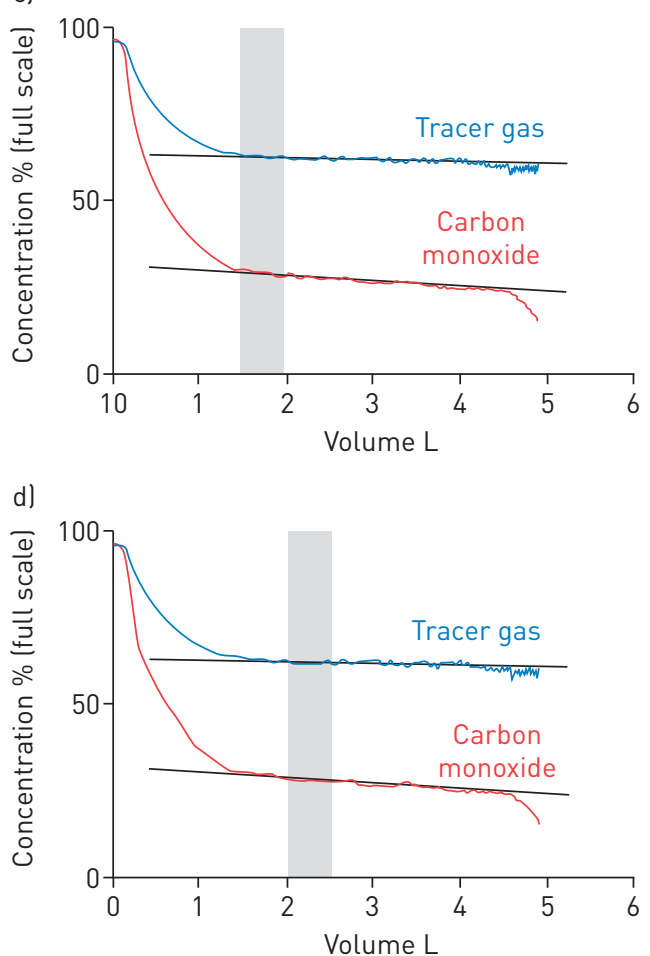

FIGURE 2 Comparison of gas concentration plotted as a function of time (a and b) or volume (c and d) for carbon monoxide and tracer gas. The shaded bar shows the collection of a $500-\mathrm{mL}$ sample of exhaled gas. The upper panels ( $a$ and $\mathrm{c}$ ) show sample collection as selected by computer algorithm (based on gas concentration and lung volume). The lower panels ( $b$ and $d$ ) show sample collection after manual adjustment by an operator using the concentration versus time plot. Operators tend to be more conservative and may over-shift the sample. When gas concentration is plotted against time, the shift does not appear to be significant; however, when gas concentration is plotted against volume, the degree of shift becomes more apparent.

samples of $85 \mathrm{~mL}$ in the development of their method and virtual alveolar gas sample volumes of $85-$ $500 \mathrm{~mL}$ may be used.

\section{Inspired gas composition}

The test gas used to calculate DLCO should contain very close to $0.3 \%$ carbon monoxide, $21 \%$ oxygen, a tracer gas and a balance of nitrogen. The tracer gas must be relatively insoluble and relatively chemically and biologically inert. The inspired carbon monoxide concentration should thus be close to $0.3 \%$. There are two factors determining the rationale for the recommendation of an inspiratory oxygen fraction $\left(F \mathrm{IO}_{2}\right)$ of $21 \%$ in the test gas for routine DLCO determination. First, the majority of studies developing reference values for DLCO, which are based on the 2005 standards [4], use an $\mathrm{FIO}_{2}$ of $21 \%$ (see the section on reference values below). Secondly, the $\mathrm{PAO}_{2}$ (alveolar oxygen tension) following a maximal inhalation will depend on the dead-space volume and the ratio of $V \mathrm{I}$ to $V \mathrm{~A}$ for any given $\mathrm{FIO}_{2}$ in the test gas. Hence if reducing $\mathrm{FIO}_{2}$ in the test gas is intended to simulate tidal breathing conditions (i.e. $\mathrm{PAO}_{2}$ of $100 \mathrm{mmHg}$ or $13 \mathrm{kPa}$ ), it may not do so in all subjects.

\section{Manoeuvre intervals}

Using an RGA system, exhaled gas can be monitored as soon as the subject begins breathing through the mouthpiece prior to the inhalation of test gas. This "end-expiratory" tracer gas information will indicate whether or not the washout from a previous manoeuvre is complete, which may occur in less than 4 min in some subjects. The tracer gas concentration at end-exhalation must be $\leqslant 2 \%$ of the tracer gas concentration in the test gas. Occasionally, if a subject has not reached this level of washout after 5 min, the operator may have the option of continuing with the next manoeuvre. However, in either event, the end-expiratory tracer gas concentration must be reported and used to compensate the tracer gas concentration data used in the determination of $V A$ at the beginning of breath-holding. 


\section{Calculations}

DLCO is calculated using the alveolar volume in litres reduced to STPD conditions (VASTPD), the breath-hold time in seconds, $t \mathrm{BH}$ (calculated by the method of JonEs and MEADE [15], the barometric pressure in $\mathrm{mmHg}, \mathrm{P}_{\mathrm{B}}$, and the fractional concentrations of carbon monoxide and tracer gas in the inspired test gas (FICO and FITr) and exhaled alveolar gas (FACO and FATr), respectively, as shown in equation 1. The conversion factor of 60000 arises from the need to convert into the correct units for DLCO $\left(\mathrm{mL} \cdot \mathrm{min}^{-1} \cdot \mathrm{mmHg}^{-1}\right)$ using $60 \mathrm{~s} \cdot \mathrm{min}^{-1}$ and $1000 \mathrm{~mL} \cdot \mathrm{L}^{-1}$.

$$
D_{\mathrm{LCO}}=\frac{V_{\mathrm{ASTPD}}}{t_{\mathrm{BH}} \cdot\left(P_{\mathrm{B}}-47\right)} \cdot \ln \left(\frac{F_{\mathrm{ICO}}}{F_{\mathrm{ACO}}} \cdot \frac{F_{\mathrm{ATr}}}{F_{\mathrm{ITr}}}\right) \cdot 60000
$$

If SI units are required, a further conversion factor of 22.4 arises from the conversion of $\mathrm{mL}(\mathrm{STPD})$ to mmol, as shown in equation 2 .

$$
T_{\mathrm{LCO}}=\frac{V_{\mathrm{ASTPD}}}{t_{\mathrm{BH}} \cdot\left(P_{\mathrm{B}}-6.28\right)} \cdot \ln \left(\frac{F_{\mathrm{ICO}}}{F_{\mathrm{ACO}}} \cdot \frac{F_{\mathrm{ATr}}}{F_{\mathrm{ITr}}}\right) \cdot 60000 / 22.4
$$

\section{Calculating the alveolar volume}

Using RGA systems, the dead-space volume is measured rather than estimated. The total dead-space, VD, can be obtained from the tracer gas washout curve using the Fowler method (figure 3) [13]. The development of RGA systems allows for the analysis of all of the exhaled gas and, since the tracer gas is being monitored throughout exhalation, there is no need to constrain the measurement of $V$ A to the discrete
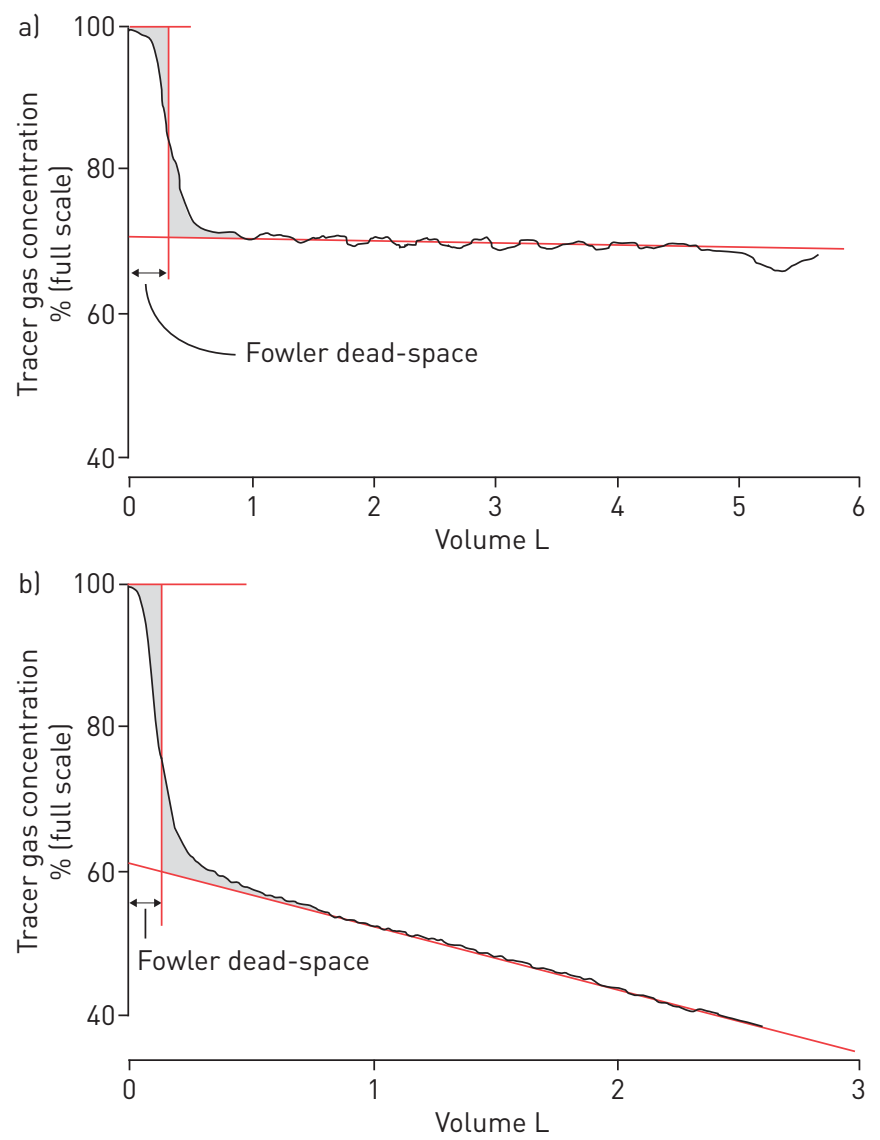

FIGURE 3 Graphical representation of the calculation of the Fowler dead-space volume in a normal, healthy subject (a) and a subject with chronic obstructive pulmonary disease (COPD) (b). The single-breath tracer gas washout is plotted against exhaled lung volume from total lung capacity. The volume at which the shaded area above the tracer gas washout curve equals the shaded area below the curve is the FowLen dead-space [13] which is reported under body temperature, ambient pressure, saturated with water vapour (BTPS) conditions. 
sample computationally constructed for determining carbon monoxide uptake. This provides the opportunity to enhance the accuracy of $V$ A determination and, indeed, using all of the available gas concentration data has been shown to provide a better estimate of $V A[16,17]$ than constraining measurement to smaller samples of exhaled gas, as required by the equipment available in 1957 when the clinical single-breath method was developed [1]

This approach uses a mass balance technique in which the volume of tracer gas inhaled and subsequently exhaled are measured, such that the volume of tracer gas remaining in the lung at end-exhalation is known $[16,17]$. The volume of tracer gas left in the lung is divided by the end-expiratory tracer gas concentration to measure the absolute end-expiratory lung volume. Total lung capacity is then calculated by adding the expired volume to the end-expiratory volume and subtracting equipment dead-space. The residual tracer gas in the lung from a previous manoeuvre, if any, can be measured prior to the start of the manoeuvre and included in the mass balance equation.

\section{RGA signal alignment}

To properly analyse continuous gas samples, the gas concentration signal from the analyser must be properly aligned with the flow signal from the pneumotachometer. The first step is to shift the concentration signal ahead in time to compensate for the lag time (figure 1). The lag time refers to the time required for the gas to travel from the aspiration port to the analyser chamber and is a function of the length and diameter of the tubing and the analyser aspiration rate. The length of the tubing should be minimised to prevent mixing of the aspirated sample within the sampling tube, which can blunt the response time through a process of Taylor dispersion. The amount of mixing will also depend on the configuration of the sampling circuit, including any valves or junctions that create turbulence. Lag time can also vary with the viscosity of the gas and as such, when helium is used as the tracer, dynamic compensation of the lag time may be required during exhalation. An additional shift of each gas concentration signal relative to the flow signal must also be performed to compensate for the response time of the analyser. This adjustment can be performed using an optimal shift equal to the natural logarithm of twice the time constant of the analyser response [18]. Alternatively, alignment may be achieved by other signal processing techniques, such as cross-correlation (convolution of signals).

\section{Evaluating the measurement of DLco Acceptability, repeatability and quality control}

The volume-time graph for a manoeuvre should show a smooth, sharp rise in volume, followed by a stable breath-hold and a smooth, sharp exhalation. The gas concentration graph should show a very sharp rise when test gas is introduced and remain stable until exhalation followed by an initial rapid decline with a smooth transition to phase III. Variations from this pattern will indicate leaks. Acceptable manoeuvres are defined in table 3. The VI of test gas must be at least $90 \%$ of the highest VC measured in the same pulmonary function testing session. At least $85 \%$ of test gas $V$ I must be inhaled $4 \mathrm{~s}$ and there must be no evidence of a Müller or Valsalva manoeuvre during the breath-hold period. The calculated breath-hold time must be $10 \pm 2 \mathrm{~s}$ and the alveolar sample collection must be completed within $4 \mathrm{~s}$. For RGA systems, the virtual sample collection must be initiated after the completion of dead-space washout. A manoeuvre with a $V \mathrm{I} / \mathrm{VC}<90 \%$ but $\geqslant 85 \%$ may be deemed acceptable if the $V \mathrm{~A}$ is within $200 \mathrm{~mL}$ or $5 \%$, whichever is greater, of another acceptable manoeuvre.

Repeatability describes the intra-session variability on repeat testing when there is no change in test conditions $[19,20]$. In a large university-based laboratory study, the coefficient of variation for repeat measurement in normal subjects was reported as $3.1 \%$ and this increased only slightly (from 4.0-4.4\%) in patients with abnormal spirometry patterns [21]. Studies conducted prior to the publication of the 2005 standards found DLCO variability of up to $9 \%$ (reproducibility) in normal individuals in repeat measurement over a period of 1 year [22] and coefficients of variation ranged from 6.2-12\% for selected UK regions [23].

Repeatability requirement: there must be at least two acceptable manoeuvres that are within $2 \mathrm{~mL} \cdot \mathrm{min}^{-1} \cdot \mathrm{mmHg}^{-1}\left(0.67 \mathrm{mmol} \cdot \mathrm{min}^{-1} \cdot \mathrm{kPa}^{-1}\right)$ of each other. A study of 4797 test sessions found that 95.5\% of cases met this criterion [24]. Since most intra-session variability is technical rather than physiological, the mean of acceptable manoeuvres is reported. The average of at least two acceptable manoeuvres that meet the repeatability requirement must be reported (i.e. outliers excluded). While it is recommended that at least two acceptable DLCO manoeuvres must be performed, research is needed to determine the actual number of manoeuvres required to provide a reasonable estimate of average DLCO for a given person. As noted elsewhere, five manoeuvres will result in an increase of $\sim 3.5 \%$ carboxyhaemoglobin $(\mathrm{COHb})$ from baseline $[25,26]$, which will decrease the measured DLCO by $\sim 3-3.5 \%$. Thus, conducting more than five manoeuvres is not a recommended strategy. 
TABLE 3 Acceptability, repeatability and quality control in DLCo testing

\section{Criteria for acceptability}

$A V_{1} \geqslant 90 \%$ of the largest $V C$ in the same test session; alternatively, a $V_{1} \geqslant 85 \%$ of the largest $V C$ in the same test

session and $V_{A}$ within $200 \mathrm{~mL}$ or $5 \%$ (whichever is greater) of the largest $V_{A}$ from other acceptable manoeuvres

$85 \%$ of test gas $V_{1}$ inhaled in $<4 \mathrm{~s}$

A stable calculated breath-hold for $10 \pm 2 \mathrm{~s}$ with no evidence of leaks or Valsalva/Müller manoeuvres during this time

Sample collection completed within $4 \mathrm{~s}$ of the start of exhalation. For RGA systems, virtual sample collection should be initiated after dead-space washout is complete

\section{Criteria for repeatability}

At least two acceptable DLco measurements within $2 \mathrm{~mL} \cdot \mathrm{min}^{-1} \cdot \mathrm{mmHg}^{-1}\left(0.67 \mathrm{mmol} \cdot \mathrm{min}^{-1} \cdot \mathrm{kPa}^{-1}\right)$ of each other

\section{Quality control grading ${ }^{\#}$}

\begin{tabular}{lccc}
\hline Score & $V_{\mathbf{I}} / \mathbf{V C}$ & $\boldsymbol{t B H}$ & Sample collection \\
\hline $\mathrm{A}$ & $\geqslant 90 \% 9$ & $8-12 \mathrm{~s}$ & $\leqslant 4 \mathrm{~s}$ \\
$\mathrm{~B}$ & $\geqslant 85 \%$ & $8-12 \mathrm{~s}$ & $\leqslant 4 \mathrm{~s}$ \\
$\mathrm{C}$ & $\geqslant 80 \%$ & $8-12 \mathrm{~s}$ & $\leqslant 5 \mathrm{~s}$ \\
$\mathrm{D}$ & $\leqslant 80 \%$ & $<8$ or $>12 \mathrm{~s}$ & $\leqslant 5 \mathrm{~s}$ \\
$\mathrm{~F}$ & $\leqslant 80 \%$ & $<8$ or $>12 \mathrm{~s}$ & $>5 \mathrm{~s}$ \\
\hline
\end{tabular}

$V_{1}$ : inspired volume; $V C$ : vital capacity; $V_{A}$ : alveolar volume; $t B H$ : breath-hold time; $D\llcorner C 0$ : diffusing capacity of the lung for carbon monoxide. " : only grade A manoeuvres meet all acceptability criteria. The average DLCO values from two or more grade A manoeuvres that meet the repeatability criterion should be reported. If only one grade A manoeuvre is attained, the DLCo value from that manoeuvre should be reported. If no grade A manoeuvre is obtained, manoeuvres of grades B to D might still have clinical utility. The average of such manoeuvres should be reported but these deviations from the acceptability criteria must be noted to caution the interpreter of the test results. Manoeuvres of grade $\mathrm{F}$ are not useable. ": or $V_{I} / \mathrm{VC} \geqslant 85 \%$ and $V_{A}$ within $200 \mathrm{~mL}$ or $5 \%$ (whichever is greater) of the largest $V_{A}$ from another acceptable manoeuvre.

There are no quality control grading systems that have been validated using the new standards contained in this document. Until such validation is published, an interim grading system is provided in table 3 and further research is recommended to develop and validate a DLCO grading system.

A grade A manoeuvre meets all acceptability criteria. The average DLCO from two or more grade A manoeuvres that are repeatable (i.e. are within $2 \mathrm{~mL} \cdot \mathrm{min}^{-1} \cdot \mathrm{mmHg}^{-1}$ or $0.67 \mathrm{mmol} \cdot \mathrm{min}^{-1} \cdot \mathrm{kPa}^{-1}$ of each other) should be reported. If, after repeat testing, the operator is unable to obtain two repeatable grade A manoeuvres, then the following values are reported with a caution to the interpreter that the testing session was suboptimal: 1) If two or more grade A manoeuvres that are not repeatable are obtained, then the average DLCO value from the acceptable manoeuvres is reported. 2) If only one grade A manoeuvre is obtained, then the DLCO value from that manoeuvre is reported. 3) If no acceptable manoeuvres are obtained, then the average DLCO value of the manoeuvres with grades B, C or D is reported. 4) If only grade F manoeuvres are obtained then no DLCO value is reported.

\section{Adjustments to the predicted value of DLco prior to interpretation}

The value of DLCO depends upon a number of physiological factors. Besides varying with age, sex, height and possibly ethnicity, DLCO also changes with lung volume, $\mathrm{Hb}$ (haemoglobin) and $\mathrm{COHb}$ level, $\mathrm{PIO}_{2}$ (inspired oxygen tension, e.g. altitude), exercise and body position.

\section{Adjustment for haemoglobin}

Using the Cotes equations [27] and assuming the "standard" $\mathrm{Hb}$ value to be $14.6 \mathrm{~g} \cdot \mathrm{dL}^{-1}$ in adult and adolescent males and $13.4 \mathrm{~g} \cdot \mathrm{dL}^{-1}$ in adult females and children $<15$ years of age, the adjustment to the predicted DLCO value of the former is described by equation 3 while the adjustment to the latter is 
described by equation 4 .

$$
\begin{aligned}
& D_{\mathrm{LCO}}[\text { predicted for } \mathrm{Hb}]=D_{\mathrm{LCO}}[\text { predicted }] \cdot(1.7 \mathrm{Hb} /(10.22+\mathrm{Hb})) \\
& D_{\mathrm{LCO}}[\text { predicted for } \mathrm{Hb}]=D_{\mathrm{LCO}}[\text { predicted }] \cdot(1.7 \mathrm{Hb} /(9.38+\mathrm{Hb}))
\end{aligned}
$$

Measurement of $\mathrm{Hb}$ in the American population [28] indicated difference from these "standard" values, especial in males, children and seniors; differences were also reported between Caucasian and African-Americans. Furthermore, the survey found that $\mathrm{Hb}$ levels in the general population were changing over time. If a more appropriate reference $\mathrm{Hb}$ level (Hbref) is available, then the predicted DLCO value is adjusted as in equation 5 .

$$
D_{\mathrm{LCO}}[\text { predicted for } \mathrm{Hb}]=D_{\mathrm{LCO}}[\text { predicted }] \cdot\left(1.7 \mathrm{Hb} /\left(0.7 \mathrm{Hb}_{\mathrm{ref}}+\mathrm{Hb}\right)\right)
$$

Adjustment for carboxyhaemoglobin concentration and carbon monoxide back-pressure

The level of $\mathrm{COHb}$ can affect the measured uptake of carbon monoxide in two ways [29-31]. First, by occupying $\mathrm{Hb}$ binding sites, carbon monoxide produces an "anaemia effect". Secondly, carbon monoxide partial pressure in the blood will reduce the driving pressure for carbon monoxide transport from alveolar gas to capillary blood.

For RGA systems, carbon monoxide back-pressure can be measured in expired gas prior to the inspiration of test gas in the DLCO manoeuvre [32] and analytically compensated for by subtracting the estimated carbon monoxide back-pressure from both the initial and final alveolar carbon monoxide partial pressures. However, this method will not adjust DLCO for the "anaemia effect" effect of COHb.

A recent study using an RGA system to measure alveolar carbon monoxide concentration combined with venous measurements of $\mathrm{COHb}$ found that the effect of carbon monoxide back-pressure and the "anaemia effect" are almost equal and the combined effect is a $2 \%$ decrease in DLCO for each $1 \%$ increase in COHb [32]. These findings were verified in a discrete-sample system [26]. In these studies, where the carbon monoxide back-pressure was measured and used in the calculation of DLCO, equation 6 was used to further correct for the anaemia effect, where FACOb is the alveolar carbon monoxide fraction in ppm measured at the end of exhalation to residual gas volume, just prior to the inhalation of test gas.

$$
D_{\mathrm{LCO}}[\text { corrected }]=D_{\mathrm{LCO}} \cdot\left(1+F_{\mathrm{ACOb}} / 560\right)
$$

\section{Adjustment of DLco for barometric pressure}

While the preceding adjustments are made to the predicted value of $D \mathrm{LCO}$, it is recommended that the measured value of DLCO be adjusted for barometric pressure. As $P \mathrm{~B}$ decreases, $\mathrm{PIO}_{2}$ decreases and DLCO increases. For factors such as $\mathrm{Hb}$ that are related to the individual subject, the recommended adjustment is made to the predicted DLCO value. However, $P_{B}$ is an environmental factor that is independent of the individual and therefore the measurement of DLCO should be adjusted to simulate standard pressure conditions. The variation in DLCO due to the typical range of high and low pressure cells at a given altitude is approximately $\pm 1.5 \%$. As mentioned above, as $P \mathrm{~B}$ decreases with altitude, DLCO increases. The rate of change is about $0.53 \%$ for each $100 \mathrm{~m}$ of increase in altitude. Moreover, the applicability of using a reference value data set from a different location is improved if both the measured DLCO and the predicted value of DLCO are adjusted to a standard pressure $(101.3 \mathrm{kPa}$ or $760 \mathrm{mmHg})$. The adjustment for $P \mathrm{~B}[3,33]$ assumes a $\mathrm{PIO}_{2}$ of $20 \mathrm{kPa}(150 \mathrm{mmHg})$ at standard pressure and the adjusted value of DLCO is thus described by equation $7(P \mathrm{~B}$ in $\mathrm{mmHg})$ or equation $8(P \mathrm{~B}$ in $\mathrm{kPa})$.

$$
\begin{aligned}
& D_{\mathrm{LCO}}\left[P_{\mathrm{B}} \text { adjusted }\right] \approx D_{\mathrm{LCO}}\left(0.505+0.00065 P_{\mathrm{B}}\right) \\
& D_{\mathrm{LCO}}\left[P_{\mathrm{B}} \text { adjusted }\right] \approx D_{\mathrm{LCO}}\left(0.505+0.00488 P_{\mathrm{B}}\right)
\end{aligned}
$$

\section{Reporting values}

This document is intended to establish technical standards which, in terms of reporting, will require DLCO systems to be able to report the variables shown in table 4 . It is not intended to specify which variables 
TABLE 4 DLCO reporting requirements

Variable

Dco (unadjusted)

DLco ladjusted for $P_{B}$ )

DLco (LLN and/or z-score)

DLco (predicted)

DLco (adjusted,predicted)

D.co $(\%$ of predicted)

$V A$ (BTPS)

VA (LLN and/or z-score)

VA (\% of predicted)

Kco

Kco (LLN and/or z-score)

Kco (predicted)

Kco $(\%$ of predicted $)$

PB

tBH

И (BTPS)

Fowler (anatomic) dead-space

TLCsb

Reference values source

Test quality grade

Operator comments

Graphs

$\mathrm{Hb}$

$\mathrm{COHb}$

Alternative calculations (e.g. three-equation

DLco, normalised slope of phase III)
Requirement

Required

Required

Required

Required

Optional (required if any adjustments

made-specify adjustments)

Required

Required

Required

Optional

Required

Required

Required

Required

Required

Required

Required

Required for RGA systems

Required for RGA systems

Required

Recommended linclude \% variability in D Lco acceptable manoeuvres)

Required (number of manoeuvres, number of acceptable manoeuvres)

Required (full manoeuvre and exhaled gas concentration versus volume with sample collection indicated for RGA systems)

Optional (required if used to adjust $D\llcorner c 0$ )

Optional (required if used to adjust $D\llcorner\mathrm{LC}$ ) Optional

BTPS: body temperature, ambient pressure, saturated with water vapour; LLN: lower limit of normal; DLCo: diffusing capacity of the lung for carbon monoxide; $V_{A}$ : alveolar volume; $K c 0$ : transfer coefficient of the lung for carbon monoxide; $P$ B: barometric pressure; $t \mathrm{BH}$ : breath-hold time; $V_{\mathrm{I}}$ (BTPS): inspired volume under BTPS conditions; VA (BTPS): alveolar volume under BTPS conditions; TLCsb: single-breath total lung capacity; $\mathrm{Hb}$ : haemoglobin; $\mathrm{COHb}$ : carboxyhaemoglobin; RGA: rapidly responding gas analyser. " : for $D \mathrm{LCO}, V_{\mathrm{A}}, K \mathrm{CO}$, $t_{B H}, V I, V$ Danat and TLCsb the average values from the acceptable and repeatable manoeuvres are reported.

end users should include in the report forms used in their laboratories, nor is it intended to address the interpretation of DLCO. Although work is ongoing towards establishing a standardised pulmonary function laboratory report form, there is no current standard. A DLCO system must be able to report the unadjusted measured DLCO, the DLCO adjusted for $P \mathrm{~B}$, the lower limit of normal and $\mathrm{z}$-score, predicted, and percentage of predicted DLCO values. They must also be able to report the carbon monoxide transfer coefficient, KCO, which is the logarithmic decay of carbon monoxide concentration over $t \mathrm{BH}$ per unit of pressure, as well as the lower limit of normal and z-score, predicted, and percentage of predicted KCO values. Any adjustments (e.g. for $\mathrm{Hb}, \mathrm{COHb}, \mathrm{PIO}_{2}$, or lung volume) must also be reported along with the data used to make the adjustment. The average VA must be reported along with the predicted VA (the predicted TLC minus the predicted $V D$ ) and percentage of predicted VA. If available, a separately measured TLC and VA/TLC ratio may optionally be reported. The average VI must also be noted. If a separately measured VC is available, it can be reported to serve as a reference for the adequacy of the VI value. In addition, comments relevant to the quality of the measurements must be included. A complete list of specifications for which variables and measurements DLCO systems should be able to report is given in table 4. While the use of $\mathrm{z}$-scores is favoured in the interpretation of pulmonary function results, given the continuing use of percentage of predicted values in many laboratories, the ability to report both $\mathrm{z}$-scores and percentage of predicted values is recommended.

The Global Lung Health Initiative (GLI) is currently working on the development of global reference values for DLCO which will very likely be of a similar structure to the GLI spirometry reference values [34]. 
Implementation of these reference values requires more complexity than simply inserting coefficients for polynomials and a DLCO system must be able to implement this method of calculating reference values.

\section{Summary}

It is not the intention of the new standards to render obsolete older equipment or instrumentation with alveolar sample chambers or bags which is still in current use. The 2005 ATS/ERS standards address this type of instrumentation and it is recognised that some equipment which meets the 2005 standards will continue to be used. However, the expectation is that new equipment will meet or exceed the new standards and that some of the systems currently available will be able to meet the new standards with software upgrades.

As already noted, the changes in DLCO standards will not impact the applicability of reference values. In general, pulmonary function measurement is more accurate and precise in normal, healthy subjects than in patients with lung disease. As such, changes which improve the measurement of DLCO will have less impact on normal, healthy subjects and will favour the continued applicability of reference values derived using older systems. There are already systematic differences amongst reference value sets for DLCO, which are related to the equipment and methodology used and which impact upon their applicability. Some reference values currently in use were developed prior to the publication of the 2005 ERS/ATS standards [3]. Hence, there is already a pressing need for reliable, comprehensive reference values for DLCO. A list of recommended research directions is given in the full standards document [2].

Advances in technology have outpaced guidelines and standards. These revisions to the DLCO standards are required to make optimal use of existing, clinically available technology. After all, guidelines and standards should not constrain progress in pulmonary function measurement but should serve to promote continual improvement.

\section{References}

1 Ogilvie C, Forster R, Blakemore W, et al. A standardized breath-holding technique for the clinical measurement of the diffusing capacity of the lung for carbon monoxide. J Clin Invest 1957; 36:1-17.

2 Graham BL, Brusasco V, Burgos F, et al. 2017 ERS/ATS Standards for single-breath carbon monoxide uptake in the lung. Eur Respir J 2017; 49: 1600016.

3 MacIntyre N, Crapo R, Viegi G, et al. Standardisation of the single-breath determination of carbon monoxide uptake in the lung. Eur Respir J 2005; 26: 720-735.

4 American Thoracic Society. Single-breath carbon monoxide diffusing capacity (transfer factor). Recommendations for a standard technique: 1995 update. Am J Respir Crit Care Med 1995; 152: 2185-2198.

5 Cotes J, Chinn D, Quanjer P, et al. Standardization of the measurement of transfer factor (diffusing capacity). Eur Respir J 1993; 6: Suppl. 16, 41-52.

6 Graham B, Mink J, Cotton D. Implementing the three-equation method of measuring single breath carbon monoxide diffusing capacity. Can Respir J 1996; 3: 247-257.

$7 \quad$ Cotes J. In Lung function. 5th Edn. London, Blackwell Scientific Publications, 1993.

8 Chinn D, Naruse Y, Cotes J. Accuracy of gas analysis in lung function laboratories. Thorax 1986; 41: $133-137$.

9 Gardner R, Clausen J, Crapo R, et al. Quality assurance in pulmonary function laboratories. ATS position paper. Am Rev Respir Dis 1986; 134: 625-627.

10 Glissmeyer E, Jensen R, Crapo R, et al. Initial testing with a carbon monoxide diffusing capacity simulator. J Invest Med 1999; 47: 37A.

11 Hegewald M, Jensen R, Teeter J, et al. Long-term intersession variability for single-breath diffusing capacity. Respiration 2012; 84: 377-384.

12 Okubo T, Lenfant C. Calibration of gas chromatograph without standardized gas mixtures. Respir Physiol 1968; 4: 255-259.

13 Fowler W. Lung function studies. II. The respiratory dead space. Am J Physiol 1948; 154: 405-416.

14 Stuart-Andrews C, Kelly V, Sands S, et al. Automated detection of the phase III slope during inert gas washout testing. J Appl Physiol 2011; 112: 1073-1081.

15 Jones R, Meade F. A theoretical and experimental analysis of anomalies in the estimation of pulmonary diffusing capacity by the single breath method. Q J Exp Physiol Cogn Med Sci 1961; 46: 131-143.

16 Graham B, Mink J, Cotton D. Effect of breath-hold time on DLCO (SB) in patients with airway obstruction. J Appl Physiol 1985; 58: 1319-1325.

17 Horstman M, Mertens F, Schotborg D, et al. Comparison of total-breath and single-breath diffusing capacity in healthy volunteers and COPD patients. Chest 2007; 131: 237-244.

18 Graham B, Buchanan P, Withy S, et al. Data acquisition from a multiplex, quadruple mass-spectrometer. Clin Phys Physiol Meas 1985; 6: 17-25.

19 Wanger J, Irvin C. Comparability of pulmonary function results from 13 laboratories in a metropolitan area. Respir Care 1991; 36: 1375-1382.

20 Gaensler E, Smith A. Attachment for automated single breath diffusing capacity measurement. Chest 1973; 63: 136-145.

21 Punjabi N, Shade D, Patel A, et al. Measurement variability in single breath diffusing capacity of the lung. Chest 2003; 123: 1082-1089.

22 Hathaway E, Tashkin D, Simmons M. Intraindividual variability in serial measurements of DLCO and alveolar volume over one year in eight healthy subjects using three independent measuring systems. Am Rev Respir Dis 1989; 140: 1818-1822.

23 Cooper B, Butterfield A. In Quality Control In Lung Function Testing. ERS Buyers' Guide To Respiratory Care Products 2009; pp. 24-38. 
24 Wise R, Teeter J, Jensen R, et al. Standardization of the single-breath diffusing capacity in a multicenter clinical trial. Chest 2007; 132: 1191-1197.

25 Frey T, Crapo R, Jensen R, et al. Diurnal variation of the diffusing capacity of the lung: is it real? Am Rev Respir Dis 1987; 136: 1381-1384.

26 Zavorsky G. The rise in carboxyhemoglobin from repeated pulmonary diffusing capacity tests. Respir Phys Neurobio 2013; 186: 103-108.

27 Cotes J, Dabbs J, Elwood P, et al. Iron-deficiency anaemia: its effect on transfer factor for the lung (diffusing capacity) and ventilation and cardiac frequency during sub-maximal exercise. Clin Sci 1972; 42: 325-335.

28 Hollowell J, Van Assendelft O, Gunter E, et al. Hematological and iron-related analytes-reference data for persons aged 1 year and over: United States, 1988-94. Vital Health Stat 2005; 11.

29 Coburn R, Forster R, Kane P. Considerations of the physiological variables that determine the blood carboxyhemoglobin concentration in man. J Clin Invest 1965; 44: 1899-1910.

30 Viegi G, Paoletti P, Carrozzi L, et al. CO diffusing capacity in a general population sample: relationship with cigarette smoking and air-flow obstruction. Respiration 1993; 60: 155-161.

31 Mohsenifar Z, Tashkin D. Effect of carboxyhemoglobin on the single breath diffusing capacity: derivation of an empirical correction factor. Respiration 1979; 37: 185-191.

32 Graham B, Mink J, Cotton D. Effects of increasing carboxyhemoglobin on the single breath carbon monoxide diffusing capacity. Am J Respir Crit Care Med 2002; 165: 1504-1510.

33 Kanner R, Crapo R. The relationship between alveolar oxygen tension and the single-breath carbon monoxide diffusing capacity. Am Rev Respir Dis 1986; 133: 676-678.

34 Quanjer P, Stanojevic S, Cole T, et al. Multi-ethnic reference values for spirometry for the 3-95-yr age range: the global lung function 2012 equations. Eur Respir J 2012; 40: 1324-1343. 